\title{
Heat current and spin current through a carbon-nanotube-based molecular quantum pump
}

\author{
Yadong Wei, ${ }^{1,2}$ Langhui Wan, ${ }^{1,2}$ Baigeng Wang, ${ }^{3,2}$ and Jian Wang ${ }^{2,4, *}$ \\ ${ }^{1}$ Department of Physics, College of Science, Shenzhen University, Shenzhen 518060, China \\ ${ }^{2}$ Department of Physics, The University of Hong Kong, Pokfulam Road, Hong Kong, China \\ ${ }^{3}$ National Laboratory of Solid State Microstructures and Department of Physics, Nanjing University, Nanjing, \\ People's Republic of China \\ ${ }^{4}$ Key Laboratory of Materials Physics, Institute of Solid State Physics, Chinese Academy of Sciences, Hefei, Anhui, \\ People's Republic of China
}

(Received 2 October 2003; revised manuscript received 26 April 2004; published 27 July 2004)

\begin{abstract}
We investigate the heat current and spin current through a carbon-nanotube-based molecular quantum pump. We have derived a general expression for the heat current at finite frequency so that the heat current can be calculated order by order in pumping amplitudes. We have applied our theory to a carbon-nanotube-based quantum pump. The heat current generated during the parametric pumping has been calculated at small frequencies for finite pumping amplitude. At finite frequencies, we have calculated the heat current to the second order in pumping amplitudes. The photon assisted process is clearly observed in the heat current. In the presence of magnetic field, the carbon-nanotube-based quantum pump can function as a spin pump, a molecular device by which a dc pure spin current without accompanying charge current is generated at zero bias voltage via a cyclic deformation of two device parameters. The pure spin current is achieved when the Fermi energy is near the resonant level of the quantum pump. We find that the pure spin current is sensitive to system parameters such as pumping amplitude, external magnetic field, and gate voltage.
\end{abstract}

DOI: 10.1103/PhysRevB.70.045418

PACS number(s): 73.63.Fg, 85.35.Kt, 72.25.Pn, 72.10.Bg

\section{INTRODUCTION}

The physics of the parametric electron pump has attracted great attention recently. ${ }^{1-37}$ When the electron motion is phase coherent and the frequency of oscillating driving forces is small compared to the inverse dwell time for carriers to traversing scattering region, the pump is considered to be an adiabatic quantum pump. ${ }^{4}$ The physical picture for such a quantum pump appears to be photon-assisted transport. ${ }^{22,26,38}$ Although the electron reservoirs are in thermal equilibrium during the pumping process, the timedependent pumping potential pumps out electrons and thus produces the Joule heat along with the dissipation at the same time. Recently, the physics of such thermal transport has been investigated. ${ }^{15,26,27,32,39}$ Avron et al. ${ }^{15}$ have derived the lower bound for the dissipation and defined an optimal pump which is noiseless. Moskalets and Buttiker ${ }^{26}$ derived a formula for the heat flow and the noise in the weak pumping regime. Wang et al. ${ }^{32}$ extended the theory to the strong pumping and finite frequency regime. They also investigated the heat current generated during pumping process for a normal-superconducting hybrid system $^{39}$ using a timedependent scattering matrix approach which goes beyond the adiabatic limit. Polianski et al. ${ }^{27}$ have studied the noise through a quantum pump for arbitrary temperatures and beyond the bilinear response.

Since the original discovery of the carbon nanotube (CNT) it has been intensively investigated in both experimental and theoretical research. ${ }^{40-48}$ CNT-based parametric electron pump has been investigated as a prototypical nanometer-scale molecular device. ${ }^{18,34}$ It would be interesting to further explore the features of heat flow through a CNT-based quantum pump. This information is also very important for device applications. In this paper, we have devel- oped a general theory for the heat current at finite frequencies based on the time-dependent scattering matrix theory. ${ }^{17,39}$ Our theory enables one to calculate the heat current at finite frequency order by order in pumping amplitude. To apply our theory in molecular devices, we investigate a parametric quantum pump that consists of a finite sized single wall carbon nanotube connected to two normal leads. Two pumping driving forces are established by applying cyclic time-dependent voltages to two metallic gates, which are capacitively coupled to the CNT. Due to these gate voltages, two potential perturbations are established along the length of the nanotube. The maximum tube length we use in this paper is up to $\sim 74 \mathrm{~nm}$. Up to the second order in pumping amplitude, we found that in the weak pumping regime the heat current increases quadratically with the pumping frequency when the frequency is small. At larger frequencies, the signature of the photon assisted process is observed in the heat current.

Since the charge and spin are two basic properties of an electron, the parametric quantum pump can also generate the spin polarized current if the external magnetic field is applied because the electron with different spins may experience different system parameters in the presence of magnetic field. It is known that the magnitude as well as the direction of the pumped current is very sensitive to various parameters of the system such as potential landscape of the pump, ${ }^{49,28}$ frequency of the driving force, ${ }^{22}$ and Fermi energy of the leads. ${ }^{52-54}$ As a consequence, it is possible to generate a spin current without accompanying charge current. Indeed, several different spin pumps have been proposed along this direction. For an interacting system, Sharma and Chamon investigated quantum pump for both spin and charge transport. ${ }^{55}$ For noninteracting systems, an adiabatic quantum pump was proposed ${ }^{56}$ which generates the spin current 
by changing the confining potential of the quantum dot and the magnetic field. In fact, this proposal has been realized experimentally. ${ }^{57}$ Spin current-including pure spin current without any charge current-was detected. A similar idea to generate spin current using the Zeeman effect has also been proposed by Aono. ${ }^{36}$ In the nonadiabatic regime, using the property of charge current reversal, the spin current can be delivered from a nonmagnetic pump connected by two ferromagnetic leads. ${ }^{58}$ The spin current can also be produced by either a rotating magnetic moment or rotating external magnetic field which leads to the unipole spin battery which gives constant spin current. ${ }^{59,60}$ To drive a spin current for future spintronic circuits, a spin-cell device which provides the necessary the constant spin-motive force is needed. ${ }^{61} \mathrm{By}$ shining microwaves on a double quantum dot in the presence of nonuniform magnetic field, such a bipolar spin battery is proposed ${ }^{61}$ In the presence of magnetic barriers, the spin current is found to pump out using the adiabatic theory. ${ }^{62} \mathrm{In}$ this paper, we explore the possibility of a carbon nanotube(CNT-) based quantum spin pump using the idea of Ref. 56. Due to the peculiar electronic properties of CNT. ${ }^{42-46}$ CNTbased quantum electron pump shows antisymmetric pumped signals near the many doubly degenerate resonant levels of the finite-length CNT for a wide range of energies. ${ }^{18}$ Because of this reversal of pumped charge current together with the Zeeman effect, ${ }^{56}$ the spin current with vanishing charge current is achieved for the Fermi energy near the resonant levels in the presence of a magnetic field. In contrast to Ref. 58, we use the external magnetic field as a tuning parameter instead of pumping frequency to achieve pure spin current and our spin pump is adiabatic. In the adiabatic regime, we numerically calculated the pumped electric and spin current using nonequilibrium Green's function method. We find that the spin current is sensitive to system parameters such as pumping amplitude, magnetic field, and gate voltage.

This paper is organized as follows. In Sec. II, based on the time-dependent scattering matrix theory, the expression of the heat current is derived. In the presence of a magnetic field, the pumped electric current and spin current are also given. In Sec. III, numerical calculations are provided for CNT based pumping devices according to our formulae and results are discussed.

\section{THEORETICAL FORMALISM FOR THE HEAT CURRENT AND SPIN CURRENT}

To calculate the heat current, we will use the timedependent scattering matrix method. ${ }^{17,39}$ We note that the heat current is defined as the particle current multiplied by the energy measured from the Fermi level. We thus have ${ }^{39}$

$$
\begin{aligned}
I_{q, \alpha}= & \frac{1}{\pi \tau} \int_{0}^{\tau} d t \int d E\left(E-E_{F}\right) \sum_{\beta} s_{\alpha \beta}(E, t) \\
& \times\left[f\left(E+i \partial_{t} / 2\right)-f(E)\right] s_{\alpha \beta}^{\dagger}(E, t) .
\end{aligned}
$$

Expanding the heat current up to $\partial_{t}^{2}$ and after some algebra, we finally obtained the heat current to $\omega^{2}$,

$$
I_{q, \alpha}^{(2)}=\frac{-1}{8 \pi \tau} \int_{0}^{\tau} d t \int d E \partial_{E} f \sum_{\beta}\left(\partial_{t} s_{\alpha \beta} \partial_{t} s_{\alpha \beta}^{\dagger}\right)
$$

which agrees with the result obtained by Moskalets and Buttiker $^{26}$ if the scattering matrix is independent of energy. To get the heat current to the order $\omega^{3}$, we need to expand Eq. (1) to $\partial_{t}^{3}$, which gives

$$
I_{q, \alpha}^{(3)}=\frac{-i}{24 \pi \tau} \int_{0}^{\tau} d t \int d E \partial_{E}^{2} f \sum_{\beta}\left(\partial_{t} s_{\alpha \beta} \partial_{t}^{2} s_{\alpha \beta}^{\dagger}\right) .
$$

Equations (2) and (3) are general expressions for the heat current low frequencies and are valid in both weak and strong pumping regimes.

To obtain the heat current at finite frequencies in the weak pumping regime, we start from the general solution for the electric current obtained by Moskalets and Buttiker ${ }^{49}$

$$
\begin{aligned}
I_{e, \alpha}= & \lim _{N->\infty} \frac{q}{N \tau} \int \frac{d E_{1}}{2 \pi} \frac{d E_{2}}{2 \pi} \sum_{\beta}\left|s_{\alpha \beta}\left(E_{1}, E_{2}\right)\right|^{2} f\left(E_{2}\right) \\
& -q \int \frac{d E}{\pi} f(E),
\end{aligned}
$$

where $2 N \tau=\int d t=2 \pi \delta(0)$. To look for a solution in the weak pumping regime, we assume that the pumping driving force at the position $x$ in the scattering region is given by

$$
V_{p p}(x)=V_{0}(x)+V_{p}(x) \cos [\omega t+\phi(x)] .
$$

Expanding the scattering matrix $s$ in terms of pumping amplitude $V_{p}$, we have

$$
s_{\alpha \beta}\left(E_{1}, E_{2}\right)=\sum_{n=0} s_{\alpha \beta}^{(n)}\left(E_{1}, E_{2}\right),
$$

where $s_{\alpha \beta}^{(n)}$ includes the $n$th order effect of pumping amplitude $\left\{V_{p}\right\}$. After some algebra, $s_{\alpha \beta}^{(n)}$ can be written as

$$
\begin{gathered}
s_{\alpha \beta}^{(0)}\left(E_{1}, E_{2}\right)=2 \pi s_{\alpha \beta}^{(0)}\left(E_{1}\right) \delta\left(E_{1}-E_{2}\right), \\
s_{\alpha \beta}^{(1)}\left(E_{1}, E_{2}\right)=2 \pi s_{+\omega, \alpha \beta}^{(1)}\left(E_{1}\right) \delta\left(E_{1}-E_{2}-\omega\right) \\
+2 \pi s_{-\omega, \alpha \beta}^{(1)}\left(E_{1}\right) \delta\left(E_{1}-E_{2}+\omega\right) .
\end{gathered}
$$

In general, we have

$$
s_{\alpha \beta}^{(n)}\left(E_{1}, E_{2}\right)=2 \pi \sum_{j=0}^{n \omega} s_{-n \omega+2 j, \alpha \beta}^{(n)}\left(E_{1}\right) \delta\left[E_{1}-E_{2}+(n \omega-2 j)\right] .
$$

We obtain

$$
\begin{aligned}
I_{e, \alpha}= & \frac{q}{N \tau} \int d E \delta(0) \sum_{\beta}\left[\left|s_{\alpha \beta}^{(0)}(E)\right|^{2} f(E)\right. \\
& +\sum_{j= \pm \omega}\left|s_{j, \alpha \beta}^{(1)}(E)\right|^{2} f(E-j)+\left[s_{\alpha \beta}^{(0)}(E) s_{0, \alpha \beta}^{(2) *}(E)+\text { c.c. }\right] f(E) \\
& \left.+\sum_{j=0, \pm 2 \omega}\left|s_{j, \alpha \beta}^{(2)}(E)\right|^{2} f(E-j)+\cdots\right]-q \int \frac{d E}{\pi} f(E) .
\end{aligned}
$$

For a time-dependent scattering matrix $s\left(t, t^{\prime}\right)$, the unitary condition reads 


$$
\int d t_{1} \sum_{\beta} s_{\alpha \beta}\left(t, t_{1}\right) s_{\alpha \beta}^{*}\left(t_{1}, t^{\prime}\right)=\delta\left(t-t^{\prime}\right)
$$

which is equivalent to

$$
\int d E_{2} /\left(4 \pi^{2}\right) \sum_{\beta} s_{\alpha \beta}\left(E_{1}, E_{2}\right) s_{\alpha \beta}^{*}\left(E_{1}, E_{2}\right)=\delta(0) .
$$

Using Eqs. (5) and (7), we find

$$
\begin{aligned}
\sum_{\beta}[ & \left|s_{\alpha \beta}^{(0)}(E)\right|^{2}+\sum_{j= \pm \omega}\left|s_{j, \alpha \beta}^{(1)}(E)\right|^{2}+\left(s_{\alpha \beta}^{(0)}(E) s_{0, \alpha \beta}^{(2)^{*}}(E)+\text { c.c. }\right) \\
& \left.+\sum_{j=0, \pm 2 \omega}\left|s_{j, \alpha \beta}^{(2)}(E)\right|^{2}+\cdots\right]=1 .
\end{aligned}
$$

Then Eq. (8) becomes

$$
\begin{aligned}
I_{e, \alpha}= & q \int \frac{d E}{\pi} \sum_{\beta}\left[\sum_{j= \pm \omega}\left|s_{j, \alpha \beta}^{(1)}(E)\right|^{2}[f(E-j)-f(E)]\right. \\
& +\sum_{j=0, \pm 2 \omega}\left|s_{j, \alpha \beta}^{(2)}(E)\right|^{2}[f(E-j)-f(E)] \\
& \left.+\sum_{j= \pm \omega}\left[s_{j, \alpha \beta}^{(1)}(E) s_{j, \alpha \beta}^{(3) *}(E)+\text { c.c. }\right][f(E-j)-f(E)]+\cdots\right] .
\end{aligned}
$$

This result is the same as that obtained by Moskalets and Buttiker $^{26}$ up to the quadratic order term, i.e., $\left|s^{(1)}\right|^{2}$ term. From this derivation, we see that if we want the quadratic order in pumping amplitude, we only need to expand $s_{\alpha \beta}$ in the linear order.

Similarly, the heat current at finite frequencies reads

$$
\begin{aligned}
I_{q, \alpha}= & \int \frac{d E}{\pi}\left(E-E_{F}\right) \sum_{\beta}\left[\sum_{j= \pm \omega}\left|s_{j, \alpha \beta}^{(1)}(E)\right|^{2}[f(E-j)-f(E)]\right. \\
& +\sum_{j=0, \pm 2 \omega}\left|s_{j, \alpha \beta}^{(2)}(E)\right|^{2}[f(E-j)-f(E)] \\
& +\sum_{j= \pm \omega}\left[s_{j, \alpha \beta}^{(1)}(E) s_{j, \alpha \beta}^{(3) *}(E)+\text { c.c. }\right] \\
& \times[f(E-j)-f(E)]+\cdots] .
\end{aligned}
$$

To analyze the quantum spin pump in the presence of a magnetic field, we consider the following Hamiltonian:

$$
H=H_{d}+H_{R}+H_{L}+H_{T}+V_{p p},
$$

where

$$
H_{\alpha}=\sum_{k, \sigma} \epsilon_{k \sigma} \hat{C}_{k \sigma \alpha}^{\dagger} \hat{C}_{k \sigma \alpha}
$$

describes the left $(\alpha=L)$ or right $(\alpha=R)$ electrodes with $\hat{C}_{k \sigma \alpha}$ the electron annihilation operator,

$$
H_{d}=\sum_{n \sigma}\left(\epsilon_{n}-\mu_{B} \sigma B\right) \hat{d}_{n \sigma}^{\dagger} \hat{d}_{n \sigma}
$$

is the Hamiltonian of the scattering region with $B$ the constant magnetic field in the CNT, and

$$
H_{T}=\sum_{n k \sigma \alpha}\left[t_{n k \sigma} \hat{C}_{k \sigma \alpha}^{\dagger} \hat{d}_{n \sigma}+\text { c.c. }\right]
$$

denotes the coupling between the scattering region and the metallic leads. Finally, $V_{p p}$ is the pumping potential. When the tight binding model of carbon atom interactions is taken into account, and the pumping driving forces $V_{p p}$ are added, the retarded Green's function can be written as

$$
G_{\sigma}^{r}(E,\{V\})=\frac{1}{E-H_{\sigma}-V_{p p}-\Sigma^{r}},
$$

where $H_{\sigma}=H_{0}-\mu_{B} \sigma B$ with $H_{0}$ describing the Hamiltonian of the CNT in the absence of the magnetic field, $\Sigma^{r} \equiv \Sigma_{L}^{r}+\Sigma_{R}^{r}$ is the total self-energy related to $t_{n k \sigma}$, and $\Gamma_{\alpha}=-2 \operatorname{Im}\left[\Sigma_{\alpha}^{r}\right]$ is the linewidth function. $V_{p p}$ is a diagonal matrix describing the variation of the CNT potential landscape due to the external pumping force $V(t)$. In this work the two external forces are chosen to be $V_{1}(t)=-V_{10}-V_{1 p} \sin (\omega t)$ and $V_{2}(t)=-V_{20}$ $-V_{2 p} \sin \left(\omega t+\phi_{0}\right)$, where $\phi_{0}$ is the phase difference between the pumping forces. In the adiabatic regime, the pumped current is zero when $\phi_{0}=0 .{ }^{4}$ However, in the nonadiabatic regime, nonzero current can be pumped out when two pumping forces are in phase. ${ }^{22}$ The potential due to the gates can therefore be written as $V_{p p}=V_{1} \Delta_{1}+V_{2} \Delta_{2}$, where $\Delta_{i}$ is the potential profile function. In the adiabatic approximation, the average pumped particle current with spin $\sigma$ through electrode $\alpha$ is given by ${ }^{4,18}$

$$
J_{\alpha \sigma}=\frac{\omega}{2 \pi} \int_{0}^{\tau} d t\left[\frac{d N_{\alpha \sigma}}{d V_{1}} \frac{d V_{1}}{d t}+\frac{d N_{\alpha \sigma}}{d V_{2}} \frac{d V_{2}}{d t}\right],
$$

where $\tau$ is the period of time-dependent pumping forces $V_{1}$ and $V_{2}$. Here the quantity $d N_{\alpha \sigma} / d V$ is the partial density of states, called the injectivity, of lead $\alpha^{63}$

$$
d N_{\alpha \sigma} / d V_{j}=-\int(d E / 2 \pi)\left(-\partial_{E} f\right) \operatorname{Tr}\left[\Gamma_{\alpha} G_{\sigma}^{r} \Delta_{j} G_{\sigma}^{a}\right]
$$

which describes the number of carriers with spin index $\sigma$ entering lead $\alpha$ due to the change of parameter $V_{j}$. Since there is no spin flipping mechanism in our system, the pumped particle current of each spin component is conserved, i.e., we have

$$
J_{L \sigma}=-J_{R \sigma}=J_{\sigma} .
$$

With Eq. (19), the pumped charge current is

$$
I_{p}=q\left(J_{\uparrow}+J_{\downarrow}\right)
$$

and the pumped spin current is $(\hbar=1)$

$$
I_{s}=\left(J_{\uparrow}-J_{\downarrow}\right) / 2 \text {. }
$$

\section{RESULTS AND DISCUSSION FOR THE HEAT CURRENT}

We first apply our theory to calculate the heat current for the system with a single wall carbon nanotube connected to two normal magnetic leads (N-CNT-N). For simplicity, the CNT is modeled with the nearest-neighbor $\pi$-orbital tight- 


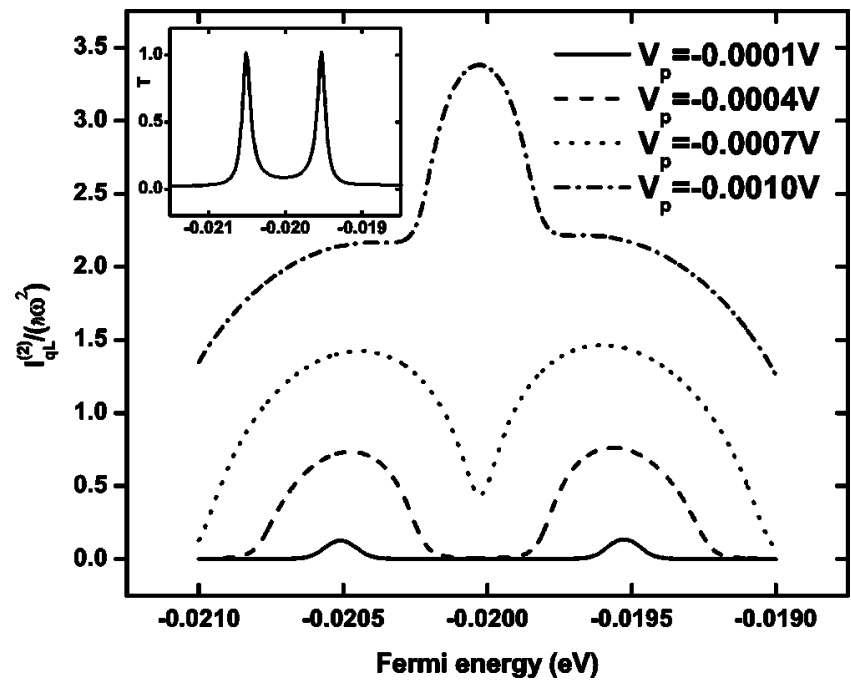

FIG. 1. The pumped heat current of the second order in pumping frequency $I_{q, L}^{(2)}$ versus Fermi energy for armchair $(7,7)$ CNT at different pumping amplitudes: $V_{p}=-0.0001 \mathrm{~V}$ (solid line), $V_{p}$ $=-0.0004 \mathrm{~V}$ (dashed line), $V_{p}=-0.0007 \mathrm{~V}$ (dotted line), and $V_{p}$ $=-0.001 \mathrm{~V}$ (dash-dotted line). Inset: transmission coefficient $T$ versus $E_{F}$ at $V_{p}=0$.

binding model with bond potential $V_{p p \pi}=-2.75 \mathrm{~V}$. This model gives a reasonable, qualitative description of the electronic and transport properties of carbon nanotubes. ${ }^{50,51} \mathrm{We}$ assume that the CNT is well contacted to the two electrodes and that both the Coulomb blockade effects and electronelectron interactions may be neglected. The two time dependent pumping driving forces are induced by the two gates near the two ends of the CNT from $0.1 L$ to $0.3 L$ and from $0.7 L$ to $0.9 L$, respectively, where $L$ is the length of the CNT. In this paper, we mimic the gate effect by simply adding the pumping potential $V_{j}$ to the CNT where the profile function $\Delta_{j}$ is set to be unity for the gate region, and zero otherwise. Hence the pumping potential can be expressed as (we suppose the symmetric pumping)

$$
V_{p p}=V_{0} \Delta+V_{p} \Delta \cos (\omega t+\phi) .
$$

In our calculation, we set $\phi=-\pi / 2$ for the first gate region and $\phi=0$ for the second gate region, so that the phase difference of the two driving forces is $\pi / 2$. A more accurate study requires a numerical solution of the Poisson equation with the gates providing the appropriate boundary conditions. In this paper, we apply the wide band limit approach in our calculation and define that the current is positive if it flows from the scattering region to reservoir. We also set $\hbar=2 \mathrm{~m}$ $=q=1$ and the temperature to be zero, i.e., do not consider the finite temperature effect.

We now consider an armchair $(7,7)$ nanotube with 600 layers of carbon atoms (total 8400 atoms). We set $V_{p}$ $=-2.74 \mathrm{~V}$ in our calculation. First, we use Eqs. (2) and (3) to calculate the heat current which gives the contribution of the second order and the third order in pumping frequencies, respectively. Figure 1 shows the heat current for the second order in pumping frequency $I_{q}^{(2)}$ versus the Fermi energy at several different pumping amplitudes. To understand this figure, the static transmission coefficient $T$ at $V_{p}=0$ as a function of the Fermi energy is also plotted in the inset of Fig. 1. One finds that there are two energy levels which give two transmission coefficient peaks in the inset. This is because the energy spectrum of the finite-sized CNT has many double degenerate energy levels. When the pumping amplitude is small, two peaks for the heat current appear near the two static energy levels and clearly show the resonant assisted behavior. When the pumping amplitude increases, the peaks of the heat current become larger and broader, and gradually approach each other. For even larger pumping amplitude, the two peaks begin to merge with additional small subpeak showing up at the center. It is interesting to compare the behavior of the pumped electric current, the heat current and the power of Joule heat. In Fig. 2 we plot the power of Joule heat $^{64}$ as the function of the Fermi energy. The pumped current as the function of the Fermi energy is shown in the inset of Fig. 2. It is easy to understand that the behavior of Joule heat is the same as that of the heat current. We see that as we

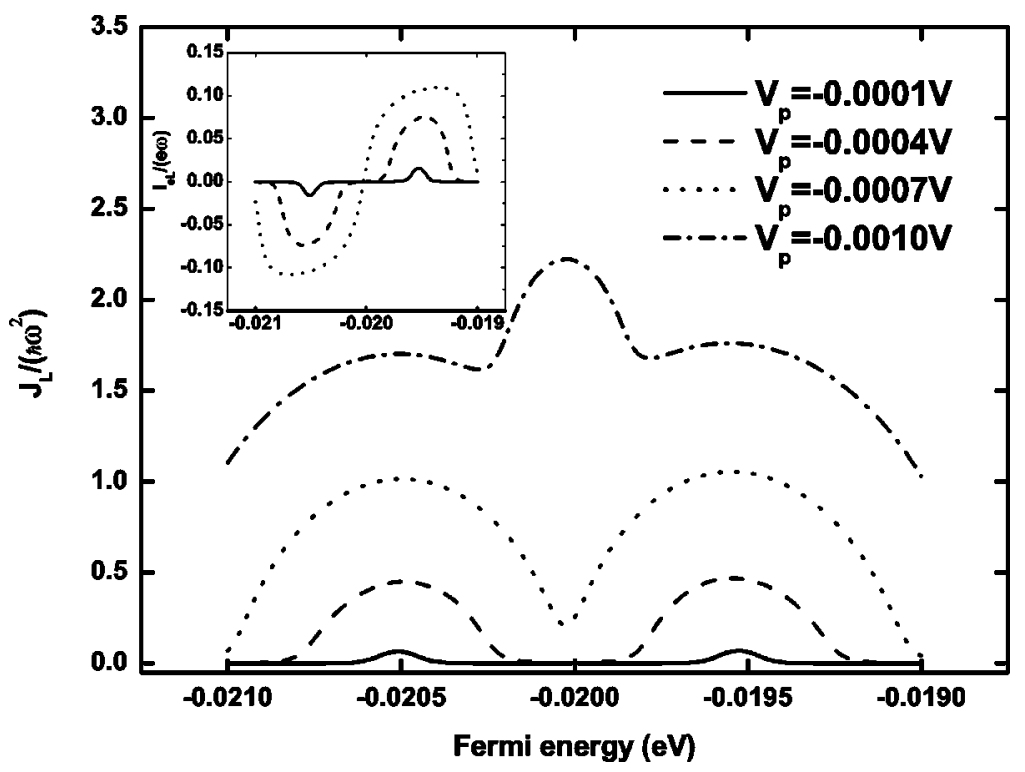

FIG. 2. The power of Joule heat of the second order in pumping frequency $J_{L}^{(2)}$ versus Fermi energy for armchair $(7,7)$ CNT at different pumping amplitudes: $V_{p}=-0.0001 \mathrm{~V}$ (solid line), $V_{p}$ $=-0.0004 \mathrm{~V}$ (dashed line), $V_{p}=-0.0007 \mathrm{~V}$ (dotted line), and $V_{p}=-0.001 \mathrm{~V}$ (dash-dotted line). Inset: The charge current of the first order in pumping frequency $I_{e}^{(1)}$ versus Fermi energy at different pumping amplitudes $V_{p}=-0.0001 \mathrm{~V}$ (solid line), $V_{p}=-0.0004 \mathrm{~V}$ (dashed line), and $V_{p}=-0.0007 \mathrm{~V}$ (dotted line). 


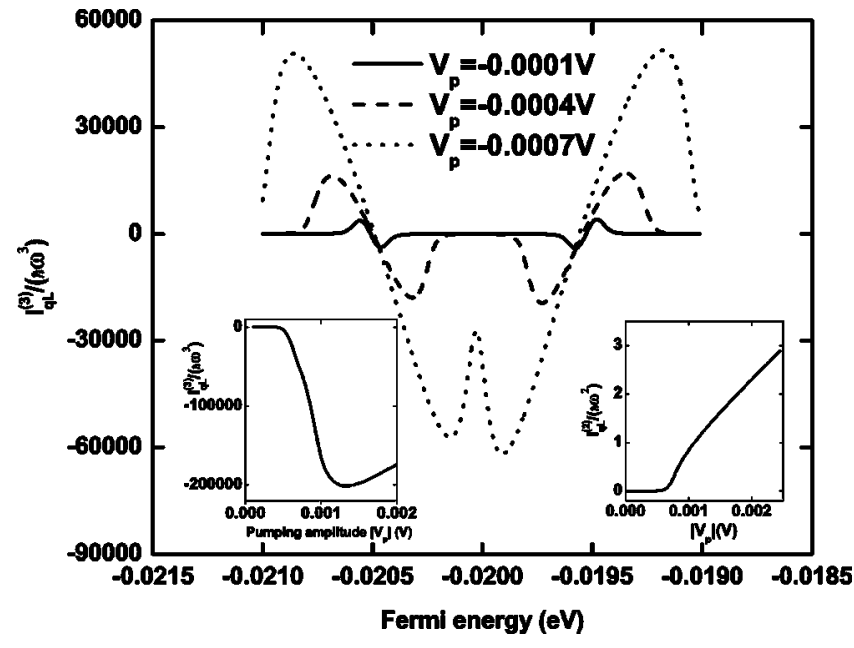

FIG. 3. The pumped heat current of the third order in pumping frequency $I_{q, L}^{(3)}$ versus Fermi energy for armchair $(7,7)$ CNT at different pumping amplitudes: $V_{p}=-0.0001 \mathrm{~V}$ (solid line), $V_{p}$ $=-0.0004 \mathrm{~V}$ (dashed line), and $V_{p}=-0.0007 \mathrm{~V}$ (dotted line). We multiplied all data by $2.11 \times 10^{16}$ and gave an additional multiple factor 5 for the solid line. Left inset: the heat current of the third order in pumping frequency $I_{q}^{(3)}$ in left lead versus pumping amplitude $V_{p}$ at $E_{f}=-0.02 \mathrm{eV}$ for armchair $(7,7) \mathrm{CNT}$. We multiplied the data by $2.11 \times 10^{16}$. Right inset: the heat current for the second order in pumping frequency $I_{q}^{(2)}$ in left lead versus pumping amplitude.

sweep the Fermi energy, the pumped current can either be positive or negative. At $E_{f} \sim-0.020037 \mathrm{eV}$, the pumped current is zero. However, exactly at this energy, the heat current and the power of Joule heat are nonzero and on the contrary reach their maximum values when the pumping amplitude is strong enough. At this energy, the quantum pump is in the most nonefficient state with maximum dissipation which one would like to avoid.

In Fig. 3, we give the heat current in the third order of pumping frequency $I_{q, L}^{(3)}$ as the function of the Fermi energy at several different pumping amplitudes. We see that $I_{q, L}^{(3)}$ can be positive or negative at different Fermi energies although $I_{q, L}^{(2)}$ flows from scattering region to reservoirs at any time. We also find that $I_{q, L}^{(3)} \sim-I_{q, R}^{(3)}$ and $I_{q, L}^{(2)} \sim I_{q, R}^{(2)}$. By analyzing data we find that at certain energies $I_{q}^{(3)^{q}}$ can reach about one percent of $I_{q}^{(2)}$ when $\omega \sim 10^{9} \mathrm{~Hz}$. While at other energies, $I_{q}^{(3)}$ is too small to be considered. In the left inset of Fig. 3, we plot $I_{q, L}^{(3)}$ as the function of pumping amplitude at $E_{f}$ $=-0.02 \mathrm{eV}$. For comparison, we also plot $I_{q, L}^{(2)}$ in the right inset of Fig. 3. We find that for armchair nanotube here, when the pumping amplitude $\left|V_{p}\right|<10^{-5} \mathrm{~V}$, the heat current is proportional to the square of the pumping amplitude $V_{p}$ and the system is in weak pumping regime. And when the pumping amplitude $\left|V_{p}\right|>10^{-3} \mathrm{~V}$, the heat current $I_{q, L}^{(2)}$ increases linearly with $V_{p}$ and the system enters into strong pumping regime. In the strong pumping region, the heat current $I_{q, L}^{(3)}$ decays gradually with the increasing of $V_{p}$.

Now we examine the quantum pump using a zigzag $(9,0)$ tube with 600 layers (total atoms 5400). We set $V_{0}=-2.3 \mathrm{~V}$ and plot the static transmission coefficient at $V_{p}=0$ as the function of Fermi energy in Fig. 4(a). We also plot the pumped current, the heat current of the second order in pumping frequency and the heat current of the third order in pumping frequency as the function of the Fermi energy in Figs. 4(b)-4(d), respectively. Very similar to the pumping behaviors in the armchair tube, we find that the behaviors of the pumped current and heat current are well correlated with the transmission coefficient of the carbon nanotube. The pumped current and heat current have large values only near the resonant levels. The charge current can flow either from the CNT to reservoirs or vice versa. The same is true of the heat current of the third order in pumping frequency. But the heat current of the second order in pumping frequency can only flow from the CNT to reservoirs. Other behaviors are similar to those of the armchair nanotube.

Finally, we investigate the heat current at finite frequencies using Eq. (13). Here, for simplicity, we only calculate the heat current to the second order in pumping amplitude $V_{p}$. We still use the armchair $(7,7)$ nanotube as an example and consider the terms including $\left|s_{ \pm \omega}^{(1)}\right|^{2}$ in Eq. (13). The numerical result is shown in Fig. 5 where we have plotted $I_{q} /\left(\omega^{2} V_{p}^{2}\right)$ vs the pumping frequency for different Fermi energies. In Fig. 5(a), we have fixed $E_{f}=-0.0205 \mathrm{eV}$ so that $E_{f}$ is in line with one of the resonant levels of the CNT: $E_{1}$ $=-0.0205 \mathrm{eV}$ and $E_{2}=-0.0195 \mathrm{eV}$ (see the inset of Fig. 1). We see that at small frequencies $\omega<10^{10} \mathrm{~Hz}$, the heat current is quadratic in pumping frequency [we have divided the heat current in Fig. 5(a) by $\left.\omega^{2}\right]$. When the pumping frequency is around $\omega \sim 1.5 \times 10^{12} \mathrm{~Hz}$, there is a peak in the heat current. This may be identified as a photon assisted process since we have $E_{2}-E_{f}=\omega$ : the electron with energy $E_{f}$ absorbs a photon and leaves the system with energy $E$ $\sim E_{2}$. To confirm this picture, in Figs. 5(b) and 5(c), we have depicted $I_{q} /\left(\omega^{2} V_{p}^{2}\right)$ vs the pumping frequency for $E_{f}$ $=-0.0210 \mathrm{eV}$ and $E_{f}=-0.0203 \mathrm{eV}$, respectively. The signature of photon assisted process is clearly seen. For instance, in Fig. 5(c), two photon assisted peaks appear at $\omega=4$ $\times 10^{11} \mathrm{~Hz}$ and $\omega=1.5 \times 10^{12} \mathrm{~Hz}$ which correspond to photon emission and absorption, respectively.

We now apply Eq. (19) to calculate the charge current and spin current for the CNT-based quantum pump. In particular, we consider a $(5,5)$ armchair CNT with 200 unit cells of carbon atoms (total 4000 atoms).

We have assumed $V_{10}=V_{20}=V_{0}$ and $V_{1 p}=V_{2 p}=V_{p}$. If the pumping is asymmetric, the transmission coefficient and hence pumped current will be suppressed. The unit of the pumped current $I_{p / s}$ is fixed by the pumping frequency. When frequency $\omega=100 \mathrm{MHz}$ which is close to the frequency used in Ref. 5, $I_{p} \sim e \omega \sim 10^{-11} \mathrm{~A}$ and $I_{s} \sim \omega / 2 \sim 10^{-8} \mathrm{eV}$ which are within experimental reach. Finally, the energy scale such as Fermi energy and gate voltages is measured in $\mathrm{eV}$. In the following calculation, we have fixed $V_{0}=2.7 \mathrm{~V}$.

In Fig. 6, we plot the pumped charge current in the absence of magnetic field when the pumping amplitude $V_{p}$ $=0.68 \mathrm{mV}$. As discussed in detail in Ref. 18, for the armchair CNT, there are many doubly degenerate levels. Near each pair of levels, the pumped charge current show patterns similar to Fig. 6. The interesting feature is that the pumped charge current reverses its direction as the Fermi energy is 


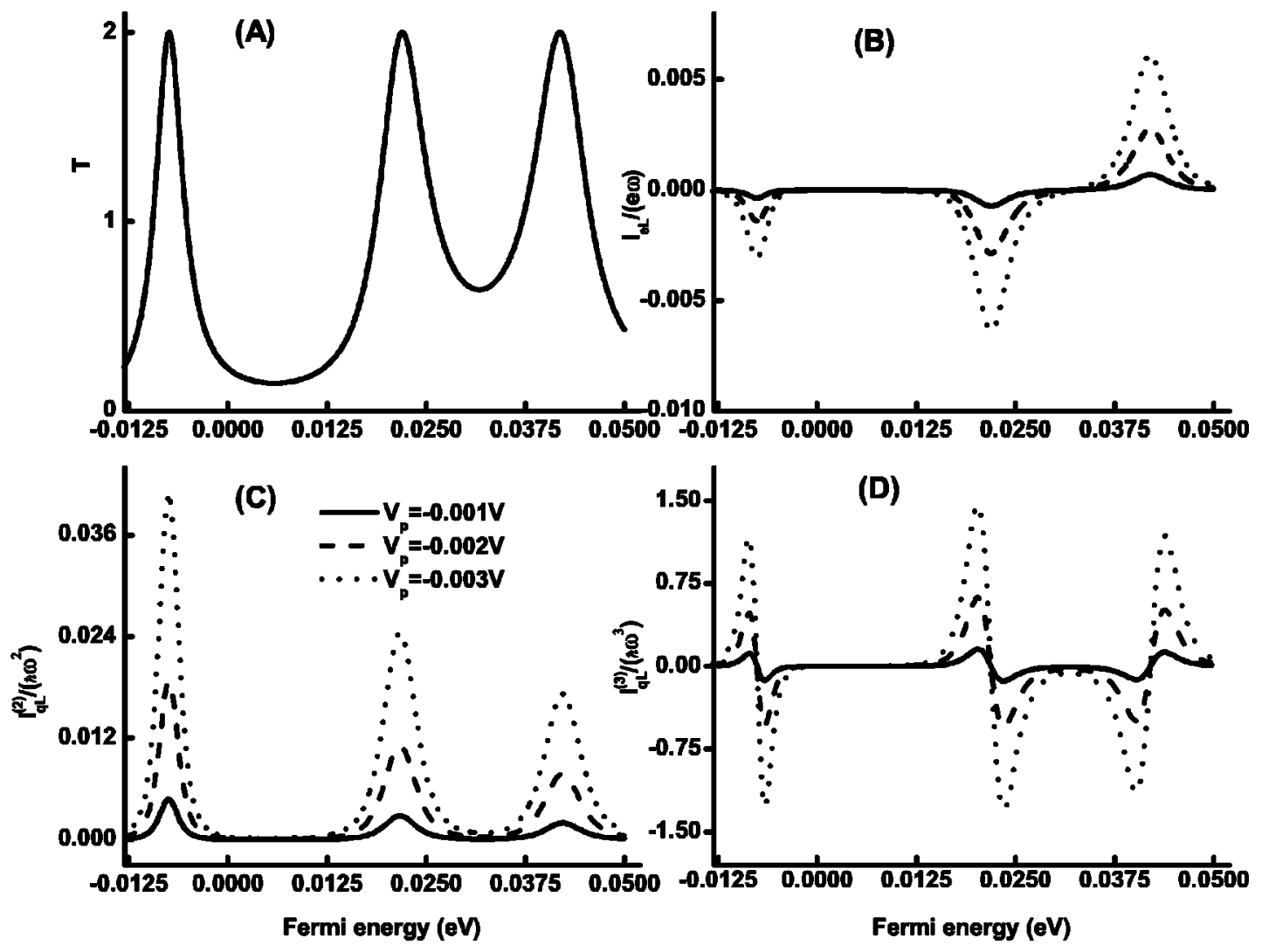

FIG. 4. (a) The static transmission coefficient versus the Fermi energy. (b) The pumped electric current versus the Fermi energy. (c) The heat current of the second order of pumping frequency versus Fermi energy. (d) The heat current of the third order of pumping frequency versus Fermi energy. Here CNT is zigzag $(9,0)$ tube with $V_{0}=-2.3 \mathrm{~V}$. In (b), (c), and (d) the solid line is for $V_{p}=-0.001 \mathrm{~V}$, the dashed line is for $V_{p}=-0.002 \mathrm{~V}$, and the dotted line is for $V_{p}=-0.003 \mathrm{~V}$.

swept which is done by changing the gate voltage. When the external magnetic field is applied, the Fermi energy of the spin up electron will be shifted downwards while for the spin down electron the shifting is in the opposite direction. Due to this Zeeman effect, the pumped spin current can be generated by tuning the magnetic field. Figure 7 shows the pumped charge current (solid line) and spin current (dotted line) as a function of the Fermi energy at a fixed magnetic field $B$ $=1.1 \mathrm{~T}^{64} \mathrm{We}$ see that the pumped charge current is zero at $E_{0}=25.4 \mathrm{meV}$. When the Fermi energy is larger than $E_{0}$ the pumped charge current is positive. As the Fermi energy is increased from $E_{0}$, the charge current rises quickly. As $E_{F}$ is

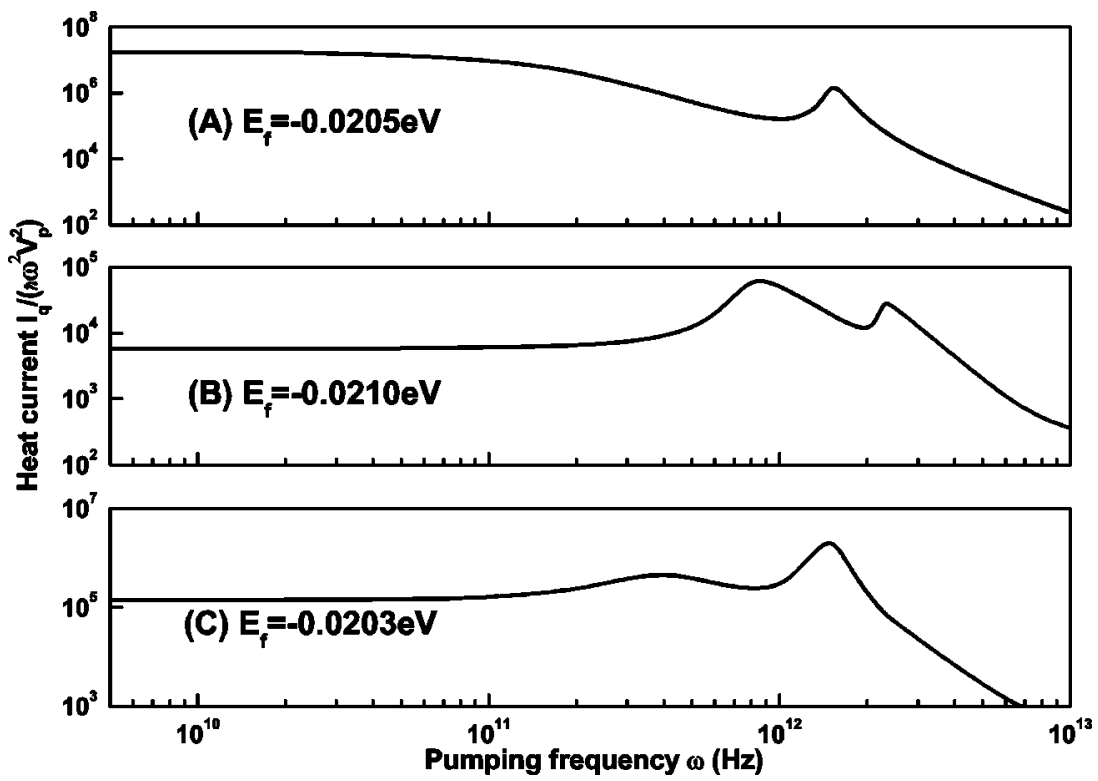

FIG. 5. The heat current of the second order of pumping amplitude versus the pumping frequency $\omega$ at different Fermi energies for an armchair $(7,7)$ CNT. We set $V_{0}=-2.74 \mathrm{eV}$. (a) $E_{f}=-0.0205 \mathrm{eV}$, $E_{f}=-0.0210 \mathrm{eV}$, $=-0.0203 \mathrm{eV}$.

(c) $E_{f}$ 


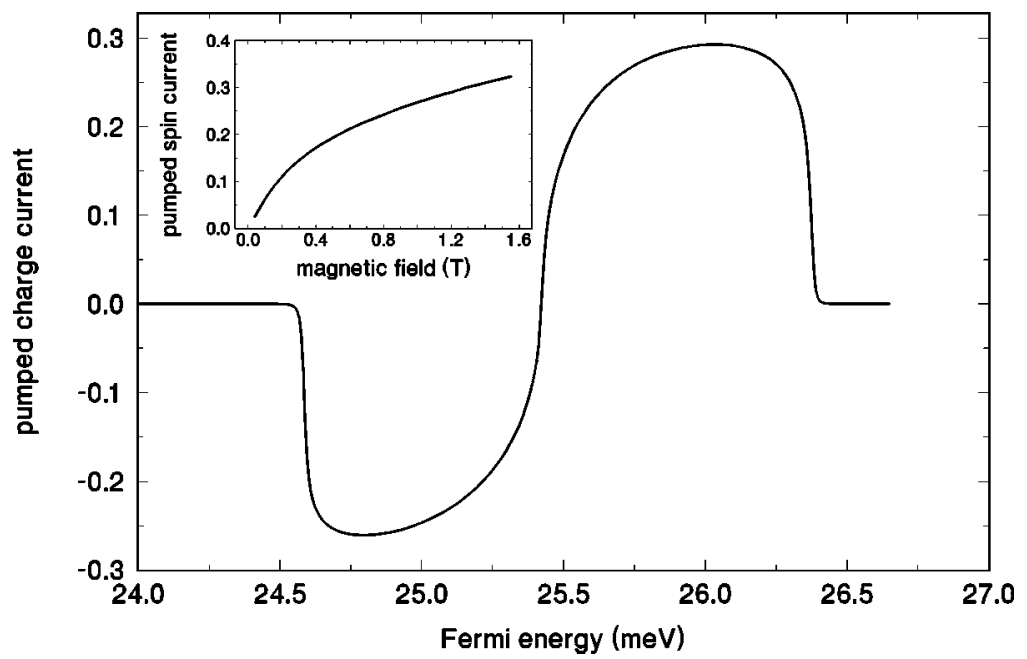

FIG. 6. The pumped charge current as a function of Fermi energy in the absence of the magnetic field. Inset: the pumped spin current as a function of magnetic field. Here $E_{F}=25.4 \mathrm{meV}$ and $V_{p}=0.68 \mathrm{meV}$, the charge current unit is $e \omega$ and the spin current unit is $\omega$.

further increased the charge current saturates and then drops quickly in the form of a double plateaulike structure. As one decreases the Fermi energy from $E_{0}$, the charge current reverses its direction. Roughly speaking, the pumped charge current is antisymmetric about the energy $E_{0}$. The pumped spin current has a positive peak at $E_{0}$ and two negative peaks near $E_{0} \pm E_{1}$ with $E_{1}=0.8 \mathrm{meV}$. At the positive peak, we have maximum spin current with vanishing charge current. We also note that the pumped spin current is in the same order of magnitude compared with the charge current. Inset of Fig. 6 depicts the pumped spin current as a function of the magnetic field when the Fermi energy is fixed at $E_{0}$. We see that the spin current increases rapidly as one turns on the magnetic field. The rate of change gradually decreases. Now we examine the spin current without accompanying charge current as a function of pumping amplitude (solid line in Fig. 8). We note that as the pumping amplitude varies, the energy at which the charge current vanishes also changes (dotted line in Fig. 8). From Fig. 8 we see that there exists a threshold for the pumping amplitude $V_{t h}=0.54 \mathrm{mV}$ above which the spin current is nonzero. Above the threshold, the pumped spin current rises quickly, peaked at $V_{p}=0.75 \mathrm{mV}$, and then decays exponentially. Finally we wish to point out that our findings are generic features of CNT quantum pump which occurs at various system parameters. For instance, in the inset of Fig. 7, we show the pumped spin current as a function of pumping amplitude when $E_{F} \approx 13.1 \mathrm{meV}$. Here we see a similar behavior compared with Fig. 8. However, the threshold for spin current and peak position are shifted to $V_{\text {th }}$ $=0.65 \mathrm{mV}$ and $V_{p}=0.94 \mathrm{mV}$, respectively. Similar physical features have also been found for other much longer $(n, n)$ nanotubes, where $(n, n)$ tube is not necessary to be $(5,5)$. To detect the spin current several methods have been proposed. (1) Due to the spin-orbit coupling the spin unpolarized current passing through a metal experiences an asymmetric scattering for electron with different spin which gives rise to the spin imbalance. Hirsch proposed ${ }^{65}$ that when a spin current passing through the same material, the charge imbalance occurs which can be used to measure the spin current. (2) The spin current induces an electric field ${ }^{66}$ which can be measured experimentally. (3) Recently, a method to measure the spin polarization of the current has been proposed and realized by Folk et al. ${ }^{68}$ using a gate-controlled bidirectional spin filter. With this technique, the measurement of spin current is experimentally feasible.

To summarize, we have investigated the heat current generated through the pumping process of a CNT-based molecular quantum pump. A general theory for the heat current at

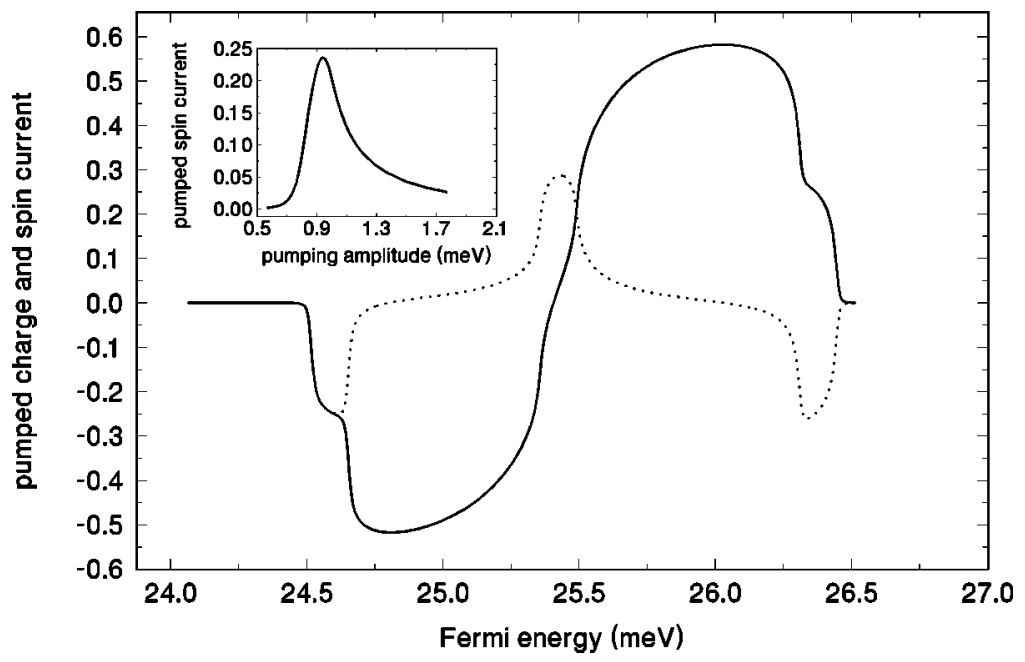

FIG. 7. The pumped charge current (solid line) and pumped spin current (dotted line) as a function of Fermi energy. Here $B=1.2 \mathrm{~T}$ and $V_{p}$ $=0.68 \mathrm{meV}$. Inset: the pumped spin current as a function pumping amplitude for $B=1 \mathrm{~T}$ and $E_{F}$ $\approx 13.1 \mathrm{meV}$. The current units are the same as those in Fig. 6. 


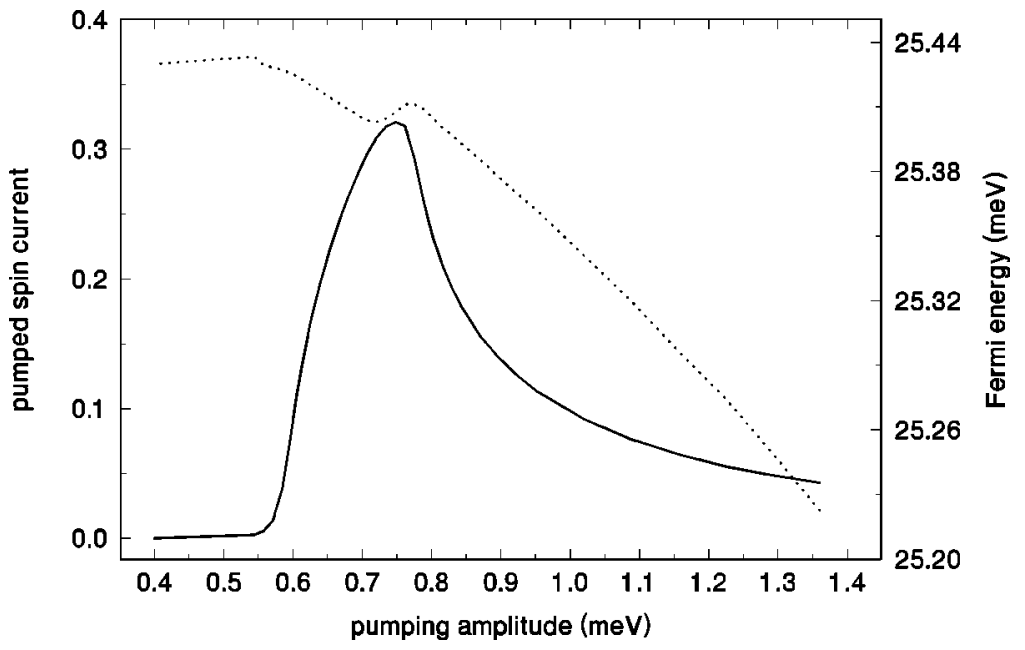

FIG. 8. The pumped spin current as a function pumping amplitude (solid line). We have also plotted the Fermi energy at which the charge current vanishes versus pumping amplitude (dotted line). Here $B=1 \mathrm{~T}$. The current units are the same as those in Fig. 6.

finite frequency is developed using the time-dependent scattering matrix approach. The theory is perturbative in nature and allows one to calculate the heat current order by order in pumping frequency. As an application, we have applied our theory to the CNT molecular quantum pump. In the adiabatic regime where the pumping frequency is small, we find that the heat flow is assisted by the resonant level in the quantum pump. The heat current of the second order in frequency always flows out of the quantum pump, while the heat current of the third order in pumping frequency can either be positive or negative. In general, the contribution to the heat current from the third order in frequency is much smaller than that from the second order in frequency. At certain Fermi energy, the pumped current is zero while the heat flow can be very large. It is also found that when the pumping frequency is smaller than $10^{10} \mathrm{~Hz}$, the heat flow is proportional to the square of pumping frequency. At larger frequencies, the photon assisted process is clearly seen in the heat current. In the presence of an external magnetic field, the quantum pump delivers a spin polarized charge current due to the Zeeman effect. The carbon nanotube based quantum pump has a unique feature that the reversal of the pumped charge current can be achieved by changing the gate voltage in the absence of the magnetic field. Due to this property together with the Zeeman effect, the spin current with zero charge current is generated near the Fermi energy that the charge current reverses its direction. In this paper, we have not considered the spin orbital effect ${ }^{67}$ which can produce a spin current without magnetic field.

\section{ACKNOWLEDGMENTS}

We gratefully acknowledge support by a RGC grant from the SAR Government of Hong Kong under Grant No. HKU 7113/02P, a CRCG grant from The University of Hong Kong, a grant from NSFC under Grant No. 10274052 (Y.D.W.), and a grant from NSFC under Grant No. 90303011 (B.G.W.).
*Electronic address: jianwang@hkusua.hku.hk

${ }^{1}$ D. J. Thouless, Phys. Rev. B 27, 6083 (1983); Q. Niu, Phys. Rev. Lett. 64, 1812 (1990).

${ }^{2}$ L. P. Kouwenhoven, A. T. Johnson, N. C. van der Vaart, C. J. P. M. Harmans, and C. T. Foxon, Phys. Rev. Lett. 67, 1626 (1991).

${ }^{3}$ H. Pothier, P. Lafarge, C. Urbina, D. Esteve, and M. H. Devoret, Europhys. Lett. 17, 249 (1992).

${ }^{4}$ P. W. Brouwer, Phys. Rev. B 58, R10 135 (1998).

${ }^{5}$ M. Switkes, C. Marcus, K. Capman, and A. C. Gossard, Science 283, 1905 (1999).

${ }^{6}$ I. L. Aleiner and A. V. Andreev, Phys. Rev. Lett. 81, 1286 (1998).

${ }^{7}$ F. Zhou, B. Spivak, and B. Altshuler, Phys. Rev. Lett. 82, 608 (1999).

${ }^{8}$ T. A. Shutenko, I. L. Aleiner, and B. L. Altshuler, Phys. Rev. B 61, 10366 (2000).

${ }^{9}$ Y. Wei, J. Wang, and H. Guo, Phys. Rev. B 62, 9947 (2000).

${ }^{10}$ I. L. Aleiner, B. L. Altshuler, and A. Kamenev, Phys. Rev. B 62,
10373 (2000).

${ }^{11}$ J. E. Avron, A. Elgart, G. M. Graf, and L. Sadun, Phys. Rev. B 62, R10 618 (2000).

${ }^{12}$ Y. Levinson, O. Entin-Wohlman, and P. Wolfle, Physica A 302, 335 (2001).

13 P. W. Brouwer, Phys. Rev. B 63, 121303 (2001); M. L. Polianski and P. W. Brouwer, ibid. 64, 075304 (2001).

${ }^{14}$ M. Moskalets and M. Buttiker, Phys. Rev. B 64, 201305 (2001).

${ }^{15}$ J. E. Avron, A. Elgart, G. M. Graf, and L. Sadun, Phys. Rev. Lett. 87, 236601 (2001).

${ }^{16}$ J. Wang, Y. D. Wei, B. G. Wang, and H. Guo, Appl. Phys. Lett. 79, 3977 (2001).

${ }^{17}$ M. G. Vavilov, V. Ambegaokar, and I. L. Aleiner, Phys. Rev. B 63, 195313 (2001).

${ }^{18}$ Y. Wei, J. Wang, H. Guo, and C. Roland, Phys. Rev. B 64, 115321 (2001).

${ }^{19}$ F. Renzoni and T. Brandes, Phys. Rev. B 64, 245301 (2001).

${ }^{20}$ Y. Makhlin and A. D. Mirlin, Phys. Rev. Lett. 87, 276803 (2001). 
${ }^{21}$ C. S. Tang and C. S. Chu, Solid State Commun. 120, 353 (2001).

${ }^{22}$ B. Wang, J. Wang, and H. Guo, Phys. Rev. B 65, 073306 (2002).

${ }^{23}$ J. Wang and B. Wang, Phys. Rev. B 65, 153311 (2002).

${ }^{24}$ O. Entin-Wohlman, Y. Levinson, and P. Wolfle, cond-mat/ 0104408 (unpublished).

${ }^{25}$ O. Entin-Wohlman, A. Aharony, and Y. Levinson, Phys. Rev. B 65, 195411 (2002).

${ }^{26}$ M. Moskalets and M. Buttiker, Phys. Rev. B 66, 035306 (2002).

${ }^{27}$ M. L. Polianski, M. G. Vavilov, and P. W. Brouwer, Phys. Rev. B 65, 245314 (2002).

${ }^{28}$ O. Entin-Wohlman and A. Aharony, Phys. Rev. B 66, 035329 (2002)

${ }^{29}$ S. L. Zhu and Z. D. Wang, Phys. Rev. B 65, 155313 (2002).

${ }^{30}$ S. W. Kim, Phys. Rev. B 66, 235304 (2002).

${ }^{31}$ B. Wang and J. Wang, Phys. Rev. B 65, 233315 (2002).

${ }^{32}$ B. Wang and J. Wang, Phys. Rev. B 66, 125310 (2002).

${ }^{33}$ M. Blaauboer, Phys. Rev. B 65, 235318 (2002).

${ }^{34}$ Y. Wei and J. Wang, Phys. Rev. B 66, 195419 (2002).

${ }^{35}$ E. R. Mucciolo, C. Chamon, and C. M. Marcus, Phys. Rev. Lett. 89, 146802 (2002).

${ }^{36}$ T. Aono, Phys. Rev. B 67, 155303 (2003).

${ }^{37}$ M. Moskalets and M. Buttiker, Phys. Rev. B 68, 161311 (2003).

${ }^{38}$ B. Wang, J. Wang, and H. Guo, Phys. Rev. B 68, 155326 (2003).

${ }^{39}$ B. Wang and J. Wang, Phys. Rev. B 66, 201305 (2002).

${ }^{40}$ S. J. Tans, M. H. Devoret, H. Dai Thess, R. E. Smalley, L. J. Geerligs, and C. Dekker, Nature (London) 386, 174 (1997).

${ }^{41}$ S. Frank, P. Poncharal, Z. L. Wang, and W. A. de Heer, Science 280, 1744 (1998).

${ }^{42}$ K. Tsukagoshi, B. W. Alphenaar, and H. Ago, Nature (London) 401, 572 (1999).

${ }^{43}$ D. H. Cobden, M. Bockrath, P. L. McEuen, A. G. Rinzler, and R. E. Smalley, Phys. Rev. Lett. 81, 681 (1998).

${ }^{44}$ Z. Yao, H. W. Ch. Postma, L. Balents, and C. Dekker, Nature (London) 402, 273 (1999).

${ }^{45}$ A. F. Morpurgo, J. Kong, C. M. Marcus, and H. Dai, Science 286, 263 (1999).

${ }^{46}$ H. Mehrez, J. Taylor, H. Guo, J. Wang, and C. Roland, Phys. Rev. Lett. 84, 2682 (2000); H. Mehrez, H. Guo, J. Wang, and C. Roland, Phys. Rev. B 63, 245410 (2001).

${ }^{47}$ C. Roland, M. Buongiorno Nardelli, J. Wang, and H. Guo, Phys. Rev. Lett. 84, 2921 (2000); C. Roland, M. Buongiorno Nardelli, H. Guo, H. Mehrez, J. Taylor, J. Wang, and Y. D. Wei, Surf. Rev. Lett. 7, 637 (2000).

${ }^{48}$ D. Orlikowski, H. Mehrez, J. Taylor, H. Guo, J. Wang, and C.
Roland, Phys. Rev. B 63, 155412 (2001).

${ }^{49}$ M. Moskalets and M. Buttiker, Phys. Rev. B 66, 205320 (2002).

${ }^{50}$ X. Blase, L. X. Benedict, E. L. Shirley, and S. G. Louie, Phys. Rev. Lett. 72, 1878 (1994); Y. A. Krotov, D. H. Lee, and S. G. Louie, ibid. 78, 4245 (1997); L. Chico, V. H. Crespi, L. X. Benedict, S. G. Louie, and M .L. Cohen, ibid. 76, 971 (1996); V. H. Crespi, M. L. Cohen, and A. Rubio, ibid. 79, 2093 (1997); L. Chico, M. P. Lopez Sancho, and M. C. Munoz, ibid. 81, 1278 (1996); L. Chico, L. X. Benedict, S. G. Louie, and M. L. Cohen, Phys. Rev. B 54, 2600 (1996).

${ }^{51}$ M. Buongiorno Nardelli, Phys. Rev. B 60, 7828 (1999).

${ }^{52}$ The power of Joule heat is defined as $(\pi / 2 \tau) \int_{0}^{\tau} d t(d Q / d t)^{2}$, where $Q=\int_{0}^{\tau} d t(d Q / d t)$ gives the pumped charge and $I_{p}=Q / \tau$ the pumped electric current. $\tau$ is the periodic time of pumping signal.

${ }^{53}$ M. Moskalets and M. Buttiker, Phys. Rev. B 66, 035306 (2002); 68, 075303 (2003).

${ }^{54}$ J. Wu, B. Wang, and J. Wang, Phys. Rev. B 66, 205327 (2002).

${ }^{55}$ P. Sharma and C. Chamon, Phys. Rev. Lett. 87, 096401 (2001).

${ }^{56}$ E. R. Mucciolo, C. Chamon, and C. M. Marcus, Phys. Rev. Lett. 89, 146802 (2002).

${ }^{57}$ S. K. Watson, R. M. Potok, C. M. Marcus, and V. Umansky, Phys. Rev. Lett. 91, 258301 (2003).

${ }^{58}$ W. Zheng, J. L. Wu, B. G. Wang, J. Wang, Q. F. Sun, and H. Guo, Phys. Rev. B 68, 113306 (2003).

${ }^{59}$ A. Brataas, Y. Tserkovnyak, G. E. W. Bauer, and B. I. Halperin, Phys. Rev. B 66, 060404 (2002).

${ }^{60}$ B. Wang, J. Wang, and H. Guo, Phys. Rev. B 67, 092408 (2003).

${ }^{61}$ Q. F. Sun, H. Guo, and J. Wang, Phys. Rev. Lett. 90, 258301 (2003).

${ }^{62}$ R. Benjamin and C. Benjamin, Phys. Rev. B 69, 085318 (2004).

${ }^{63}$ M. Büttiker and T. Christen, in Quantum Transport in Semiconductor Submicron Structures, edited by B. Kramer (Kluwer Academic Publishers, Dordrecht, 1996), p. 263.

${ }^{64}$ In the experiment of Ref. 57, $B=4 \mathrm{~T}$ was used.

${ }^{65}$ J. E. Hirsch, Phys. Rev. Lett. 83, 1834 (1999).

${ }^{66}$ F. Meier and D. Loss, Phys. Rev. Lett. 90, 167204 (2003); Q. F. Sun, H. Guo, and J. Wang, Phys. Rev. B 69, 054409 (2004).

${ }^{67}$ M. Governale, F. Taddei, and R. Fazio Phys. Rev. B 68, 155324 (2003); P. Sharma and P. W. Brouwer, Phys. Rev. Lett. 91, 166801 (2003).

${ }^{68}$ J. A. Folk, R. M. Potok, C. M. Marcus, and V. Umansky, Science 299, 679 (2003). 